\title{
Theoretical stability and materials synthesis of a chemically ordered MAX phase, Mo2ScAIC2, and its two-dimensional derivate Mo2ScC2 MXene
}

Rahele Meshkian, Quanzheng Tao, Martin Dahlqvist, Jun Lu, Lars Hultman and Johanna Rosén

\section{Journal Article}

\section{Tweet}

N.B.: When citing this work, cite the original article.

Original Publication:

Rahele Meshkian, Quanzheng Tao, Martin Dahlqvist, Jun Lu, Lars Hultman and Johanna Rosén, Theoretical stability and materials synthesis of a chemically ordered MAX phase, Mo2ScAlC2, and its two-dimensional derivate Mo2ScC2 MXene, Acta Materialia, 2017. 125(), pp.476-480.

$\underline{\text { http://dx.doi.org/10.1016/j.actamat.2016.12.008 }}$

Copyright: Elsevier

http://www.elsevier.com/

Postprint available at: Linköping University Electronic Press

http://urn.kb.se/resolve?urn=urn:nbn:se:liu:diva-136312
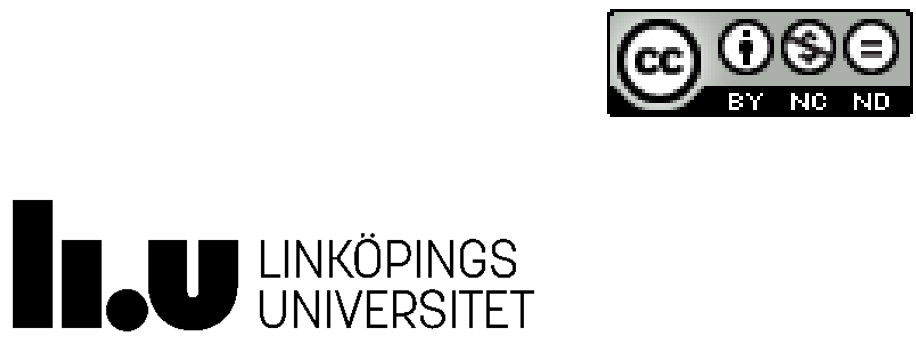
Theoretical stability and materials synthesis of a chemically ordered MAX phase, $\mathrm{Mo}_{2} \mathrm{ScAlC}_{2}$, and its two-dimensional derivate $\mathrm{Mo}_{2} \mathrm{ScC}_{2} \mathrm{MXene}$

Rahele Meshkian*, Quanzheng Tao, Martin Dahlqvist, Jun Lu, Lars Hultman, and Johanna Rosen

Department of Physics, Chemistry and Biology (IFM), Linköping University, SE-581 83 Linköping, Sweden

\begin{abstract}
We present theoretical prediction and experimental evidence of a new MAX phase alloy, $\mathrm{Mo}_{2} \mathrm{ScAlC}_{2}$, with out-of-plane chemical order. Evaluation of phase stability was performed by ab initio calculations based on Density Functional Theory, suggesting that chemical order in the alloy promotes a stable phase, with a formation enthalpy of -24 meV/atom, as opposed to the predicted unstable $\mathrm{Mo}_{3} \mathrm{AlC}_{2}$ and $\mathrm{Sc}_{3} \mathrm{AlC}_{2}$. Bulk synthesis of $\mathrm{Mo}_{2} \mathrm{ScAlC}_{2}$ is achieved by mixing elemental powders of $\mathrm{Mo}$, Sc, $\mathrm{Al}$ and graphite which are heated to $1700{ }^{\circ} \mathrm{C}$. High resolution transmission electron microscopy reveals a chemically ordered structure consistent with theoretical predictions with one Sc layer sandwiched between two Mo-C layers. The two-dimensional derivative, the MXene, is produced by selective etching of the Al-layers in hydrofluoric acid, resulting in the corresponding chemically ordered $\mathrm{Mo}_{2} \mathrm{ScC}_{2}$, i.e. the first Sc-containing MXene. The here presented results expands the attainable range of MXene compositions and widens the prospects for property tuning.
\end{abstract}

Keywords: laminated structure, out-of-plane chemical order, MAX phase, 2D material; MXene, DFT calculations

*Corresponding author; Tel. +4613286619, e-mail address: rahele.meshkian@liu.se 


\section{Introduction}

It has been about six decades since Nowotny et al. discovered a family of laminated material called $H$-phases [1]. After their revival by Barsoum et al. some decades later [2], the family was expanded and given the nomenclature $M_{n+1} A X_{n}$ (MAX) phases, $n=1-3$, being composed of an early transition metal $(M)$, an A-group element primarily from group 13 and $14(A)$, and carbon and/or nitrogen $(X)$. These compounds are inherently laminated, and exhibit a combination of metallic and ceramic properties which stem from strong metallic-covalent $M$ $X$ bonds in combination with weaker bonding between $M-A$ atoms. Consequently, MAX phases display high electrical and thermal conductivity, good resistance to oxidation and thermal shock, and are elastically stiff and easily machinable. To date, more than 70 MAX phases have been synthesized in both bulk and thin film form.

Substitution of a fraction of $M, A$, or $X$ atoms can be beneficial for property tuning, e.g., for increasing the hardness [3], or for introducing magnetic properties [4, 5]. MAX phase alloys to date are to a major extent solid solutions, and in particular alloys of $211(n=1)$ stoichiometry have not shown any tendency to order in atomic layers composed of one element only, possibly due to a high configurational entropy within these systems and only one crystallographic site for each M, A, and X element [6]. This is opposed to quaternary MAX phases of $312(n=2)$ or $413(n=3)$ stoichiometry and with $M$-site alloying, which can display an out-of-plane chemical order. Such examples are the recently reported $\mathrm{Mo}_{2} \mathrm{TiAlC}_{2}$ and $\mathrm{Mo}_{2} \mathrm{Ti}_{2} \mathrm{AlC}_{3}$, which were theoretically predicted and subsequently synthesized by Anasori et al. [7]. This is in addition to previously discovered $\mathrm{Cr}_{2} \mathrm{TiAlC}_{2}$ and $\mathrm{V}_{1.5} \mathrm{Cr}_{1.5} \mathrm{AlC}_{2}$, reported by Liu et al. [8] and Caspi et al. [9], respectively. Note that for $\mathrm{V}_{1.5} \mathrm{Cr}_{1.5} \mathrm{AlC}_{2}$, a 
partially ordered structure has been observed. In an explanatory and predictive theoretical study by Dahlqvist et al. [6], the authors have investigated the stability of TiMAlC, $\mathrm{Ti}_{2} \mathrm{AlC}_{2}, M \mathrm{Ti}_{2} \mathrm{AlC}_{2}$, and $\mathrm{Ti}_{2} M_{2} \mathrm{AlC}_{3}$ where $M$ is from group 4-6 in the Periodic table of elements, trying to identify the origin behind the chemical ordering. Extending beyond that study, exploring a combination of $M$ elements that can neither be found in a pure 312 MAX phase nor energetically promote a stacking in which $M$ is surrounded by $\mathrm{C}$ in an face-centered cubic (fcc) configuration, we have here investigated quaternary MAX phases in the Mo-ScAl-C system.

Interest in Al-containing MAX phases increased after evidence of their resistance to oxidation upon formation of protective oxide layers [10,11], also used in studies focused towards crack healing $[12,13]$. Moreover, selective etching of Al has been shown to produce MXenes, graphene analogous materials that are both electrically conducting and hydrophilic [14]. The quest for Mo-containing MXenes in particular was elevated after a number of theoretical studies, predicting these compounds as promising thermoelectric material [15], as catalyst [16] and also as efficient electrodes for Li-ion batteries [17]. The first $\mathrm{Mo}_{2} \mathrm{C}$ MXene was reported in 2015 [18, 19], and has since been found to have high potential for e.g. energy storage, in particular for electrode material in e.g. Li-ion batteries [20].

There is only one previous report stating synthesis of a Sc-based MAX phase; $\mathrm{Sc}_{2} \operatorname{InC}[1$, 21]. However, no information is presented on the specific synthesis conditions, and no experimental evidence of the resulting material or its properties. There is a theoretical report on the structural and elastic properties of a number of known and hypothetical $M_{2} \operatorname{InC}$ phases 
with $M=\mathrm{Sc}, \mathrm{Ti}, \mathrm{V}, \mathrm{Nb}, \mathrm{Zr}, \mathrm{Hf}$ and Ta. Beside the calculated crystal parameters, the authors have reported the theoretical Young's, shear, and bulk moduli, which for $\mathrm{Sc}_{2} \mathrm{InC}$ are well below the other phases investigated [22].

Consequently, exploring synthesis of a MAX phase based on Al, Mo and Sc is highly motivated from a fundamental as well as a property perspective. In the present study, we have theoretically predicted and experimentally verified the existence of $\mathrm{Mo}_{2} \mathrm{ScAlC}_{2}$ as a new chemically ordered MAX phase. Structural and compositional characterization show separation of the elements into individual atomic layers. Furthermore, we present evidence of the corresponding MXene; $\mathrm{Mo}_{2} \mathrm{ScC}_{2}$.

\section{Computational details}

First-principles calculations were performed by means of density functional theory (DFT) and the projector augmented wave method $[23,24]$ as implemented within the Vienna $a b$ initio simulation package (VASP) [25-27]. We adopted the non-spin polarized generalized gradient approximation (GGA) as parameterized by Perdew-Burke-Ernzerhof (PBE) [28] for treating electron exchange and correlation effects. A plane-wave energy cut-off of $400 \mathrm{eV}$ was used and for sampling of the Brillouin zone we used the Monkhorst-Pack scheme [29]. For each considered phase the calculated total energy is converged to within $0.5 \mathrm{meV} / \mathrm{atom}$ with respect to $k$-point sampling and structurally optimized in terms of unit-cell volumes, $c / a$ ratios (when necessary), and internal parameters to minimize the total energy. 
Chemically disordered structures denote a solid solution of Sc and Mo on the $M$-sites. These are modelled using the special quasi-random structure (SQS) method [30, 31] on supercells of $4 \times 4 \times 1 M_{3} A X_{2}$ unit cells, with a total of $96 M$-sites, respectively. Convergence tests with respect to total energy show that these sizes are appropriate to use, based on an energy of the $4 \times 4 \times 1$ unit cells being within 2 meV/atom compared to larger supercells.

Evaluation of phase stability was performed by identifying the set of most competing phases at a given composition, using a linear optimization procedure [31, 32] including all competing phases in the system. A phase is considered thermodynamically stable when its energy is lower than the set of most competing phases, and when there is no imaginary frequencies in phonon spectra, i.e. an indicated dynamic stability. The approach has been proven successful to confirm already experimentally known MAX phases as well as to predict the existence of new ones [7, 33, 34].

When the temperature $T \neq 0 \mathrm{~K}$, Gibbs free energy of a disordered phase, $\Delta G_{\mathrm{cp}}^{\text {disorder }}$, can be approximated using

$$
\Delta G_{\mathrm{cp}}^{\text {disorder }}=\Delta H_{\mathrm{cp}}^{\text {disorder }}-T \Delta S
$$

Where $\Delta H_{\mathrm{cp}}^{\text {disorder }}$ is the formation enthalpy and $\Delta S$ is the entropy per formula unit of an ideal solution of Sc and Mo atoms on the $M$-sites, expressed as

$$
\Delta S=-y k_{B}[z \ln (z)+(1-z) \ln (1-z)]
$$

where $y$ is number of $M$-sites per formula unit, i.e., $y=n+1$, and $z=\mathrm{Mo} /(\mathrm{Sc}+\mathrm{Mo})$.

Moreover, when $T \neq 0 \mathrm{~K}$, the configurational entropy $\Delta S$ will decrease the free energy, 
$\Delta G_{\mathrm{cp}}^{\text {disorder }}$, for the solid solution. By using Eq. 1 and 2, an order-disorder temperature $T_{\text {disorder }}$ can be calculated for which $\Delta G_{\mathrm{cp}}^{\text {disorder }}[T]=\Delta H_{\mathrm{cp}}^{\text {order }}$. This gives an estimate above which temperature the disordered structure is energetically favorable as compared to the ordered structure. The temperature can then be compared to the experimental conditions used, e.g., typical bulk synthesis temperatures of 1200 - $1600{ }^{\circ} \mathrm{C}(1473$ - $1873 \mathrm{~K})$.

\section{Experimental details}

Elemental powders of graphite (99.999\%), Mo (99.99\%), (Sigma-Aldrich), Sc (99.99\%, Stanford Advanced Material), Al (99.8\%, ALFA AESAR) with mesh sizes of -200, -400, 200 and -200, respectively, were used for the materials synthesis. These powders were mixed in an agate mortar and placed in a covered $\mathrm{Al}_{2} \mathrm{O}_{3}$ crucible, which was inserted in a tube furnace. This was heated at a rate of $10{ }^{\circ} \mathrm{C}$ per minute up to $1700{ }^{\circ} \mathrm{C}$, where it was kept for 30 minutes, with a resulting total duration of $4 \mathrm{~h}$. After cooling down to room temperature in the furnace, the sintered sample was crushed and mixed, resulting in a fine $\mathrm{Mo}_{2} \mathrm{ScAlC}_{2}$ powder.

Structural characterization was performed by X-ray diffraction (XRD) on a diffractometer (Rikagu Smartlab, Tokyo, Japan), with $\mathrm{Cu}-\mathrm{K}_{\alpha}$ radiation (40 kV and $44 \mathrm{~mA}$ ). $\theta-2 \theta$ scans were recorded between $3^{\circ}$ and $120^{\circ}$ and step scans of $0.02^{\circ}$ with a step size of 7 s. The scan was analyzed by Rietveld refinement using FULLPROF code [35, 36]. The fitting parameters used in the program were 5 backgrounds parameters, scale factor, $X$ and $Y$ profile parameter to limit the peak width to the major phase, lattice parameters, atomic positions and 
occupancies for all phases, and overall B-factor for the main phase. Both MAX and MXene samples were also characterized by using the Linköping double $\mathrm{C}_{\mathrm{s}}$ corrected FEI Titan3 60300 operated at $300 \mathrm{kV}$, equipped with the Super-X EDX system to perform atomic structural analysis.

For MXene preparation, 1 gram of the fine powder was added to $20 \mathrm{ml}$ of $48 \%$ aqueous hydrofluoric acid (HF), and kept at $50{ }^{\circ} \mathrm{C}$ for a duration of $\sim 16 \mathrm{~h}$. The suspension was afterwards filtered and dispersed in water $~ 10$ times in order to remove all the remaining acid and the reaction products. Subsequent intercalation of the MXene sheets were realized by adding $\sim 0.1 \mathrm{~g}$ of the powder in $\sim 1 \mathrm{ml}$ of an organic base, tetrabutylammonium hydroxide (TBAOH), and shaking it manually for $\sim 5 \mathrm{~min}$. After centrifuging the solution for 5 min the remaining TBAOH can be washed away using water, a procedure repeated 2-3 times.

\section{Results and discussion}

For $\mathrm{Mo}_{2} \mathrm{ScAlC}_{2}$ and $\mathrm{Sc}_{2} \mathrm{MoAlC}_{2}$, six different layer sequences were considered, see Anasori et al. [37] for layer stacking definitions. In addition, a solid solution of Sc and Mo on the Msites was also taken into account, see Data in Brief Table 1.

Only $\mathrm{Mo}_{2} \mathrm{ScAlC}_{2}$ of order A, i.e., with Sc at Wyckoff site 2a and Mo at site 4f, is predicted stable at $0 \mathrm{~K}$ with a calculated formation enthalpy of -24 meV/atom. A complete list of considered competing phases used for the phase stability evaluation can be found in Data in Brief Table 2. Furthermore, $T_{\text {disorder }}=4031 \mathrm{~K}$, indicating that ordered structures are expected at typical synthesis temperatures. Note that neither $\mathrm{Sc}_{3} \mathrm{AlC}_{2}$ nor $\mathrm{Mo}_{3} \mathrm{AlC}_{2}$ are 
stable, with calculated $\Delta H_{\mathrm{cp}}=+155$ and $+141 \mathrm{meV} /$ atom, respectively. For $\mathrm{Sc}_{2} \mathrm{MoAlC}_{2}$ none of the considered layer sequences nor the disordered structure are predicted stable. This is further supported in analysis of dynamical stability, i.e. stability with respect to lattice vibrations, through calculated phonon spectra, see Data in Brief Figure $1 . \mathrm{Mo}_{2} \mathrm{ScAlC}_{2}$ is found to be dynamically stable, while both $\mathrm{Mo}_{3} \mathrm{AlC}_{2}$ and $\mathrm{Sc}_{3} \mathrm{AlC}_{2}$ are dynamically unstable with distinct imaginary phonon modes.

Fig. 1 shows the measured XRD scan (red), the Rietveld refinement (black) with refinement parameters listed in Data in Brief Table 3, and the difference between the two former scans (blue). The calculated Bragg positions for $\mathrm{Mo}_{2} \mathrm{ScAlC}_{2}(\sim 73.9 \mathrm{wt} \%)$ and the impurity phases, $\mathrm{Mo}_{2} \mathrm{C}$ (14.2 wt\%), $\mathrm{Al}_{2} \mathrm{O}_{3}$ (7.4 wt\%), $\mathrm{Mo}_{3} \mathrm{Al}_{2} \mathrm{C}$ ( $\left.3.5 \mathrm{wt} \%\right)$, and $\mathrm{Mo}_{3} \mathrm{Al}(\sim 1.0 \mathrm{wt} \%)$ is shown with the vertical lines. The in-plane and out-of-plane lattice parameters, $a$ and $c$, determined from Rietveld refinement, are 3.03 and $18.77 \AA$, respectively. The theoretical values are slightly higher, 3.0619 and $19.072 \AA$, respectively, which is common when using GGA functionals.

The optimized starting ratios of Mo:Sc:Al:C powders were 2:1.05:1:2. The 5\% extra Sc was added to compensate for loss of Sc during the synthesis process.

Structural characterization from HRSTEM and local elemental mapping for the $\mathrm{Mo}_{2} \mathrm{ScAlC}_{2}$ powder is shown in Fig. 2. A highly ordered and laminated arrangement of the phase along the $[11 \overline{2} 0]$ zone axis is apparent in Fig. 2(a). The EDX elemental mapping of the Mo, Sc and Al within the sample is illustrated in Fig. 2(b), where the Sc layers (green) are clearly interleaved in between two layers of Mo (red) which, in turn, is bonded to Al. Compositional 
analysis shows that the relative concentrations of Mo, Sc and $\mathrm{Al}$ are 57, 19 and 23 at\%, respectively, with an estimated error bar of $\pm 5 \%$. The slight deviation from the ideal 2:1:1 ratio of Mo:Sc:Al originating from a higher Mo content may be explained by partial intermixing between the $\mathrm{M}$ elements, as expected from entropic reasons and based on observations from previously identified chemically ordered 312 phases [7, 9] and from Rietveld refinement suggesting Mo potentially occupying a few of the Sc positions, as also indicated in Fig. 2(b).

The stability resulting from out-of-plane chemical ordering in some quaternary MAX phases has been suggested to originate from a specific $\mathrm{M}$ element (e.g. Ti) breaking an energetically unfavorable stacking of another $\mathrm{M}$ element surrounded by $\mathrm{C}$ in an face-centered cubic (fcc) configuration [6, 7]. Furthermore, if the $\mathrm{M}$ element closest to the Al layer has larger electronegativity than $\mathrm{Al}$, this results in fewer electrons available for populating antibonding Al-Al orbitals, which is energetically expensive [6]. Applying these arguments for the present material, $\mathrm{Mo}_{2} \mathrm{ScAlC}_{2}$, it is correspondingly not energetically favorable for Mo atoms to be surrounded by $\mathrm{C}$ atoms in a fcc configuration. However, this is valid also for Sc, i.e. opposed to $\mathrm{Ti}$, the Sc atoms cannot break an unfavorable stacking. The explanation for the stability of $\mathrm{Mo}_{2} \mathrm{ScAlC}_{2}$ must therefore be found elsewhere, possibly in the electronic configurations due to the large difference in electronegativity between Sc (1.36) and Mo (2.16). Sc, which is at Wyckoff position 2a, may give away electrons to C, which has a very high electronegativity (2.55). Mo, which has a higher electronegativity than Al (1.61), will likely attract electrons from the Al layer. The latter is supported by Bader analysis (see Data in Brief Figure 2), 
showing that $\mathrm{Al}$ is positively charged in $\mathrm{Mo}_{2} \mathrm{ScAlC}_{2}$ as well as in the hypothetical $\mathrm{Mo}_{3} \mathrm{AlC}_{2}$, though not in the hypothetical $\mathrm{Sc}_{3} \mathrm{AlC}_{2}$.

Turning to the 2D derivative of this novel alloy, the general MXene formula can be written as $M_{n+1} X_{n} T_{x}$, where $T_{x}$ denotes surface functionalization, though we here only use the common notation $M_{n+1} X_{n}$. X-ray diffractograms of the $\mathrm{Mo}_{2} \mathrm{ScAlC}_{2} \mathrm{MAX}$ powder and its corresponding MXene, $\mathrm{Mo}_{2} \mathrm{ScC}_{2}$, after etching and intercalation, is shown in Fig. 3. A new peak corresponding to MXene appears around 7.14 degrees after HF etching (increasing $c$ lattice parameter (LP) to $24.6 \AA$ ), which shifts to 5.06 degrees after intercalation (increasing $c$ to $34.8 \AA$ ). The etching is not fully completed as the scan for the MXene also contains residual $\mathrm{Mo}_{2} \mathrm{ScAlC}_{2}$.

Micrographs from HRSTEM analysis of the sample after etching are shown in Fig. 4. An overview micrograph is shown in (a), for which there is no $\mathrm{Al}$ remaining, i.e. the region is fully converted to MXene. A higher resolution image is shown in (b), where the EDX elemental mapping clearly shows Mo as well as Sc in the MXene. The Mo-Sc-Mo chemical order is weakly indicated from the line scan, however, elemental mapping with atomic resolution is challenging for the MXene due to non-flat sheets of the material. It should be noted that Sc-F-containing particles can be found in the sample, suggesting that Sc may be partially etched and bonding to $\mathrm{F}$ in the acid solution. We suggest that this may be possible due to a slight Mo-Sc intermixing, as mentioned above, where the Sc atoms residing within the outer Mo-layers will contact the acid and hence possibly be etched. This partial etching 
of Sc is consistent with local composition analysis from the EDX mapping in Fig. 4(b), showing a Mo:Sc ratio of 79:21.

The here presented work is the first report on a Sc-containing MAX phase since the claimed existence of $\mathrm{Sc}_{2} \mathrm{InC}$ by Toth et al. 1966 [21]. More important is the finding of a Sc-based MXene. To date, MXenes like $\mathrm{Mo}_{2} \mathrm{C}$ [18] as well as $\mathrm{Mo}_{2} \mathrm{Ti}$ and $\mathrm{Mo}_{2} \mathrm{Ti}_{2}$ [37] are known, and the present contribution therefore increases the potential for property tuning of Mo-based MAX phases as well as their corresponding MXenes.

\section{Conclusions}

We have theoretically predicted the existence of a new quaternary MAX phase alloy with out-of-plane chemical order, $\mathrm{Mo}_{2} \mathrm{ScAlC}_{2}$, with a Sc atomic layer sandwiched between two Mo-C layers. The prediction has been experimentally verified through bulk synthesis and materials characterization, primarily from high resolution STEM with EDX elemental mapping. The $a$ and $c$ lattice parameters determined using Rietveld refinement are 3.03 and 18.77 A, respectively. Furthermore, the MAX phase has been converted into twodimensional MXene by selective etching of Al. The resulting MXene, $\mathrm{Mo}_{2} \mathrm{ScC}_{2}$, is the first MXene to date comprising Sc. 


\section{Acknowledgement}

J. R. acknowledges funding from the Swedish Research Council (VR) under grant no. 6212012-4425 and 642-2013-8020, from the the Knut and Alice Wallenberg (KAW) Foundation, and from the Swedish Foundation for Strategic Research (SSF) through the synergy grant FUNCASE. L.H. acknowledges the KAW Foundation for a Scholar Grant and support to the Linköping Ultra Electron Microscopy Laboratory. The simulations were carried out using supercomputer resources provided by the Swedish National Infrastructure for Computing (SNIC) at the National Supercomputer Centre (NSC), the High Performance Computing Center North (HPC2N), and the PDC Center for High Performance Computing. J.Halim is acknowledged for XRD measurements.

\section{References}

[1] H. Nowotny. Strukturchemie einiger verbindungen der übergangsmetalle mit den elementen C, Si, Ge, Sn, Prog. Solid. State. Chem, 5 (1971) 27-70.

[2] M.W. Barsoum. MAX Phases: Properties of Machinable Ternary Carbides and Nitrides, WileyVCH, Weinheim, 2013.

[3] F.L. Meng, Y.C. Zhou, J.Y. Wang. Strengthening of Ti2AlC by substituting Ti with V, Scripta Materialia 53 (2005) 1369-1372.

[4] M. Dahlqvist, B. Alling, I.A. Abrikosov, J. Rosen. Magnetic nanoscale laminates with tunable exchange coupling from first principles, Physical Review B 84 (2011) 220403-220405.

[5] A. Mockute, M. Dahlqvist, J. Emmerlich, L. Hultman, J.M. Schneider, P.O.Å. Persson, J. Rosen. Synthesis andab initiocalculations of nanolaminated (Cr,Mn $)_{2} \mathrm{AlC}$ compounds, Physical Review B 87 (2013) 094113-094114.

[6] M. Dahlqvist, J. Rosen. Order and disorder in quaternary atomic laminates from first-principles calculations, Phys. Chem. Chem. Phys. 17 (2015) 31810-31821.

[7] B. Anasori, M. Dahlqvist, J. Halim, E.J. Moon, J. Lu, B.C. Hosler, E.a.N. Caspi, S.J. May, L. Hultman, P. Eklund, J. Rosén, M.W. Barsoum. Experimental and theoretical characterization of ordered MAX phases $\mathrm{Mo}_{2} \mathrm{TiAlC}_{2}$ and $\mathrm{Mo}_{2} \mathrm{Ti}_{2} \mathrm{AlC}_{3}$, J. Appl. Phys. 118 (2015) 094304-094314. [8] Z. Liu, E. Wu, J. Wang, Y. Qian, H. Xiang, X. Li, Q. Jin, G. Sun, X. Chen, J. Wang, M. Li. Crystal structure and formation mechanism of $\left(\mathrm{Cr}_{2 / 3} \mathrm{Ti}_{1 / 3}\right)_{3} \mathrm{AlC}_{2}$ MAX phase, Acta Materialia 73 (2014) 186-193. 
[9] E.a.N. Caspi, P. Chartier, F. Porcher, F. Damay, T. Cabioc'h. Ordering of (Cr,V) Layers in Nanolamellar $\left(\mathrm{Cr}_{0.5} \mathrm{~V}_{0.5}\right)_{\mathrm{n}+1} \mathrm{AlC}_{\mathrm{n}}$ Compounds, Materials Research Letters 3 (2014) 100-106.

[10] M. Sundberg, G. Malmqvist, A. Magnusson, T. El-Raghy. Alumina forming high temperature silicides and carbides, Ceramics International 30 (2004) 1899-1904.

[11] Q.M. Wang, A. Flores Renteria, O. Schroeter, R. Mykhaylonka, C. Leyens, W. Garkas, M. to

Baben. Fabrication and oxidation behavior of $\mathrm{Cr}_{2} \mathrm{AlC}$ coating on Ti6242 alloy, Surface and Coatings Technology 204 (2010) 2343-2352.

[12] H.J. Yang, Y.T. Pei, J.C. Rao, J.T.M. De Hosson. Self-healing performance of $\mathrm{Ti}_{2} \mathrm{AlC}_{\text {ceramic, }}$ Journal of Materials Chemistry 22 (2012) 8304-83013.

[13] G.M. Song, Y.T. Pei, W.G. Sloof, S.B. Li, J.T.M. De Hosson, S. van der Zwaag. Oxidationinduced crack healing in $\mathrm{Ti}_{3} \mathrm{AlC}_{2}$ ceramics, Scripta Materialia 58 (2008) 13-16.

[14] M. Naguib, J. Come, B. Dyatkin, V. Presser, P.-L. Taberna, P. Simon, M.W. Barsoum, Y. Gogotsi. MXene: a promising transition metal carbide anode for lithium-ion batteries,

Electrochemistry Communications 16 (2012) 61-64.

[15] M. Khazaei, M. Arai, T. Sasaki, M. Estili, Y. Sakka. Two-dimensional molybdenum carbides: potential thermoelectric materials of the MXene family, Phys. Chem. Chem. Phys. 16 (2014) 78417849.

[16] T. Matsumoto, Y. Nagashima, T. Yamazaki, J. Nakamura. Fuel Cell Anode Composed of $\mathrm{Mo}_{2} \mathrm{C}$ Catalyst and Carbon Nanotube Electrodes, Electrochemical and Solid-State Letters 9 (2006) A160-A162.

[17] H.-J. Zhang, K.-X. Wang, X.-Y. Wu, Y.-M. Jiang, Y.-B. Zhai, C. Wang, X. Wei, J.-S. Chen. $\mathrm{MoO}_{2} / \mathrm{Mo}_{2} \mathrm{C}$ Heteronanotubes Function as High-Performance Li-Ion Battery Electrode, Advanced Functional Materials 24 (2014) 3399-3404.

[18] R. Meshkian, L.-Å. Näslund, J. Halim, J. Lu, M.W. Barsoum, J. Rosen. Synthesis of twodimensional molybdenum carbide, $\mathrm{Mo}_{2} \mathrm{C}$, from the gallium based atomic laminate $\mathrm{Mo}_{2} \mathrm{Ga}_{2} \mathrm{C}$, Scripta Materialia 108 (2015) 147-150.

[19] C. Xu, L. Wang, Z. Liu, L. Chen, J. Guo, N. Kang, X.L. Ma, H.M. Cheng, W. Ren. Large-area high-quality 2D ultrathin Mo2C superconducting crystals, Nat Mater 14 (2015) 1135-1141.

[20] J. Halim, S. Kota, M.R. Lukatskaya, M. Naguib, M.-Q. Zhao, E.J. Moon, J. Pitock, J. Nanda, S.J. May, Y. Gogotsi, M.W. Barsoum. Synthesis and Characterization of 2D Molybdenum Carbide (MXene), Advanced Functional Materials 26 (2016) 3118-3127.

[21] L. E. Toth, W. Jeitschko, C.M. Yen. The superconducting behavior of several complex carbides and nitrides, Journal of the Less Common Metals 10 (1966) 29-32.

[22] A. Bouhemadou. Calculated structural and elastic properties of $\mathrm{M}_{2} \operatorname{InC}(\mathrm{M}=\mathrm{Sc}, \mathrm{Ti}, \mathrm{V}$, Zr,Nb,Hf,Ta), Modern Physics Letters B 22 (2008) 2063-2076.

[23] P.E. Blöchl. Projector augmented-wave method, Physical Review B 50 (1994) 17953-17979.

[24] G. Kresse, D. Joubert. From ultrasoft pseudopotentials to the projector augmented-wave method, Phys. Rev. B 59 (1999) 1758-1775.

[25] G. Kresse, J. Hafner. Ab initio molecular dynamics for liquid metals, Phys. Rev. B 47 (1993) 558-561.

[26] G. Kresse, J. Furthmüller. Efficiency of ab-initio total energy calculations for metals and semiconductors using a plane-wave basis set, Comput. Mater. Sci. 6 (1996) 15-50.

[27] G. Kresse, J. Furthmüller. Efficient iterative schemes for ab initio total-energy calculations using a plane-wave basis set, Phys. Rev. B 54 (1996) 11169-11186.

[28] J.P. Perdew, K. Burke, M. Ernzerhof. Generalized gradient approximation made simple, Phys. Rev. Lett. 77 (1996) 3865-3868. 
[29] H.J. Monkhorst, J.D. Pack. Special points for Brillouin-zone integrations, Phys. Rev. B 13 (1976) 5188-5192.

[30] A. Zunger, S.H. Wei, L.G. Ferreira, J.E. Bernard. Special quasirandom structures, Phys. Rev. Lett. 65 (1990) 353-356.

[31] M. Dahlqvist, B. Alling, I.A. Abrikosov, J. Rosén. Phase stability of $\mathrm{Ti}_{2} \mathrm{AlC}$ upon oxygen incorporation: A first-principles investigation, Physical Review B 81 (2010) 024111-024118.

[32] M. Dahlqvist, B. Alling, J. Rosén. Stability trends of MAX phases from first principles, Physical Review B 81 (2010) 220102-220104.

[33] P. Eklund, M. Dahlqvist, O. Tengstrand, L. Hultman, J. Lu, N. Nedfors, U. Jansson, J. Rosen. Discovery of the ternary nanolaminated compound $\mathrm{Nb} 2 \mathrm{GeC}$ by a systematic theoreticalexperimental approach, Phys. Rev. Lett. 109 (2012) 035502-035504.

[34] A.S. Ingason, A. Mockute, M. Dahlqvist, F. Magnus, S. Olafsson, U.B. Arnalds, B. Alling, I.A. Abrikosov, B. Hjorvarsson, P.O. Persson, J. Rosen. Magnetic self-organized atomic laminate from first principles and thin film synthesis, Phys. Rev. Lett. 110 (2013) 195502-195505.

[35] H.M. Rietveld. A Profile Refinement Method for Nuclear and Magnetic Structures, Journal of Applied Crystallography 2 (1969) 65-71.

[36] J. Rodriguez-Carvajal. Recent advances in magnetic structure determination by neutron powder diffraction, Physica B 192 (1993) 55-69.

[37] B. Anasori, Y. Xie, M. Beidaghi, J. Lu, B. C. Hosler, L. Hultman, P. R. C. Kent, Y. Gogotsi, M. W. Barsoum. Two-Dimensional, Ordered, Double Transition Metal Carbide (MXene), Journal of American Chemical Society Nano 9 (2015) 9507-9516.

[38] V.L. Deringer, A.L. Tchougréeff, R. Dronskowski. Crystal Orbital Hamilton Population (COHP) Analysis As Projected from Plane-Wave Basis Sets, The Journal of Physical Chemistry A 115 (2011) 5461-5466.

[39] R. Dronskowski, P.E. Bloechl. Crystal orbital Hamilton populations (COHP): energy-resolved visualization of chemical bonding in solids based on density-functional calculations, The Journal of Physical Chemistry 97 (1993) 8617-8624.

[40] S. Maintz, V.L. Deringer, A.L. Tchougréeff, R. Dronskowski. Analytic projection from planewave and PAW wavefunctions and application to chemical-bonding analysis in solids, Journal of Computational Chemistry 34 (2013) 2557-2567. 


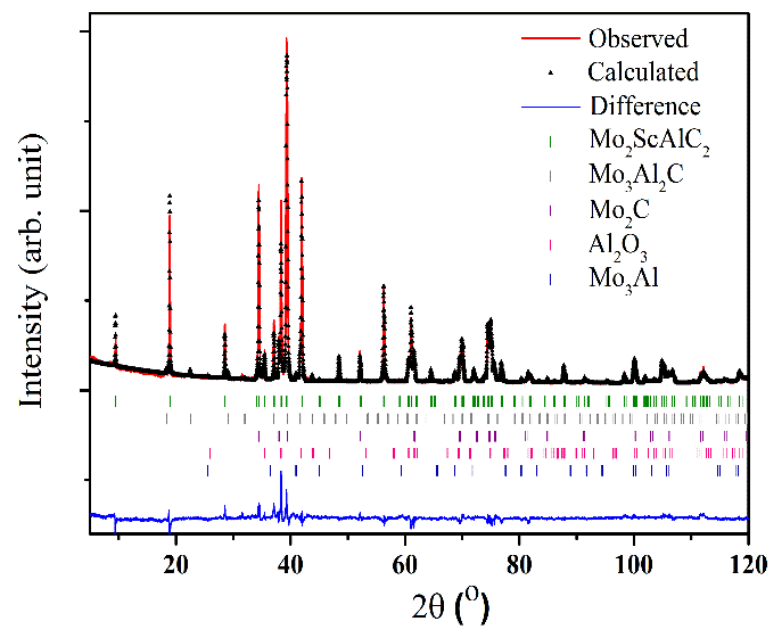

Fig. 1. XRD analysis; measured scan (red), and the calculated pattern (black) using Rietveld refinement. The blue line is the difference between the two. The Bragg positions for the main phase, $\mathrm{Mo}_{2} \mathrm{ScAlC}_{2}$ (top) and the included phases, $\mathrm{Mo}_{3} \mathrm{Al}_{2} \mathrm{C}, \mathrm{Mo}_{2} \mathrm{C}, \mathrm{Al}_{2} \mathrm{O}_{3}$ and $\mathrm{Mo}_{3} \mathrm{Al}$ can also be seen in the figure. 


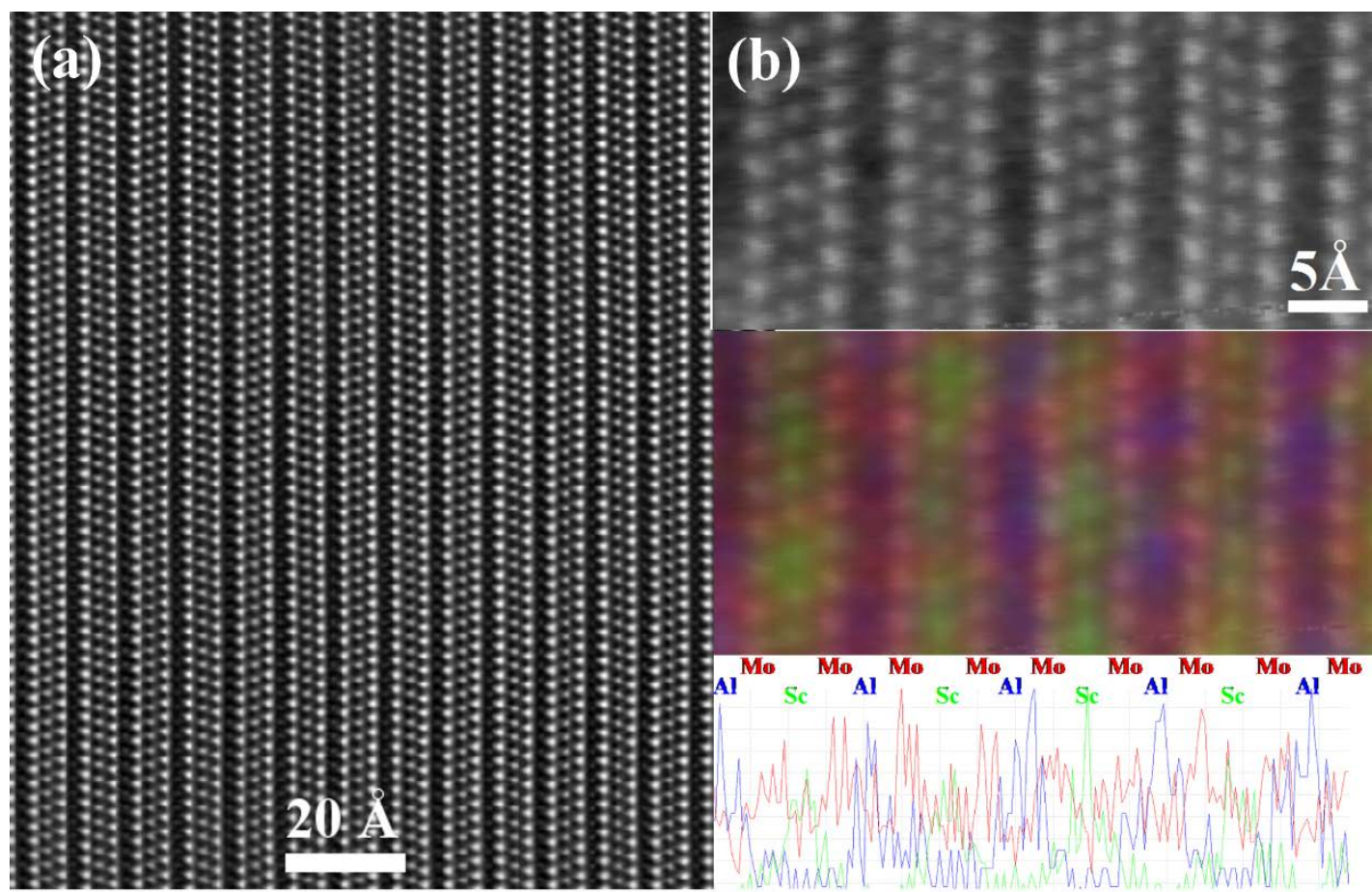

Fig. 2. (a) HR(S)TEM micrograph shows the laminated structure of the MAX phase along the [11 $\overline{2} 0]$ zone axis. (b) The overlapping EDX elemental map for Mo, Sc and $\mathrm{Al}$ reveals the chemically ordered distribution of these elements within the sample. 


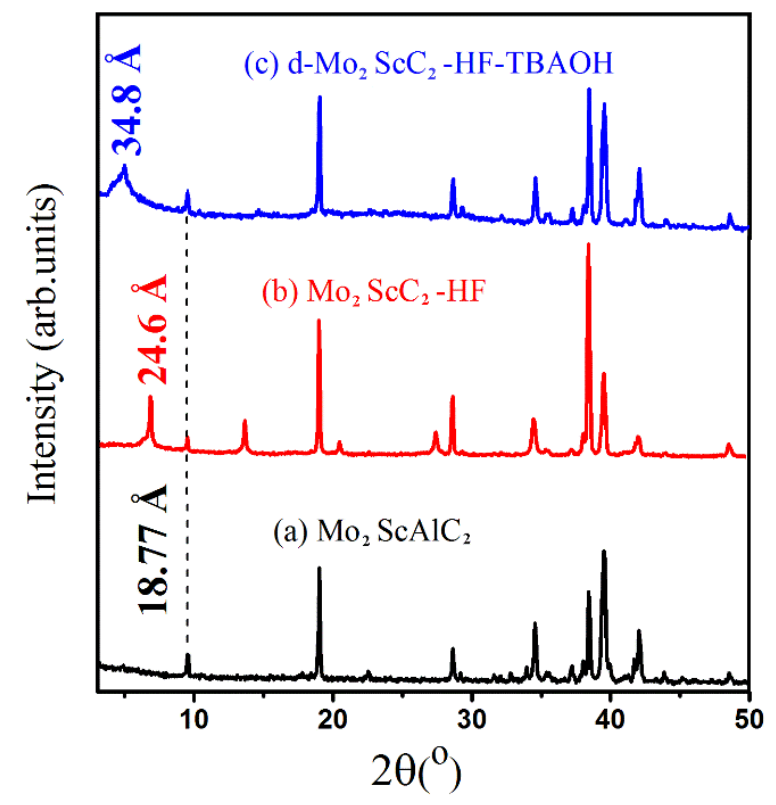

Fig. 3. XRD scan of (a) $\mathrm{Mo}_{2} \mathrm{ScAlC}_{2} \mathrm{MAX}$ powder (b) $\mathrm{Mo}_{2} \mathrm{ScC}_{2} \mathrm{MXene}$, and (c) MXene after intercalation in TBAOH solution. The $c$ lattice parameters for these samples are 18.77, 24.6 and $34.8 \AA$, respectively. 


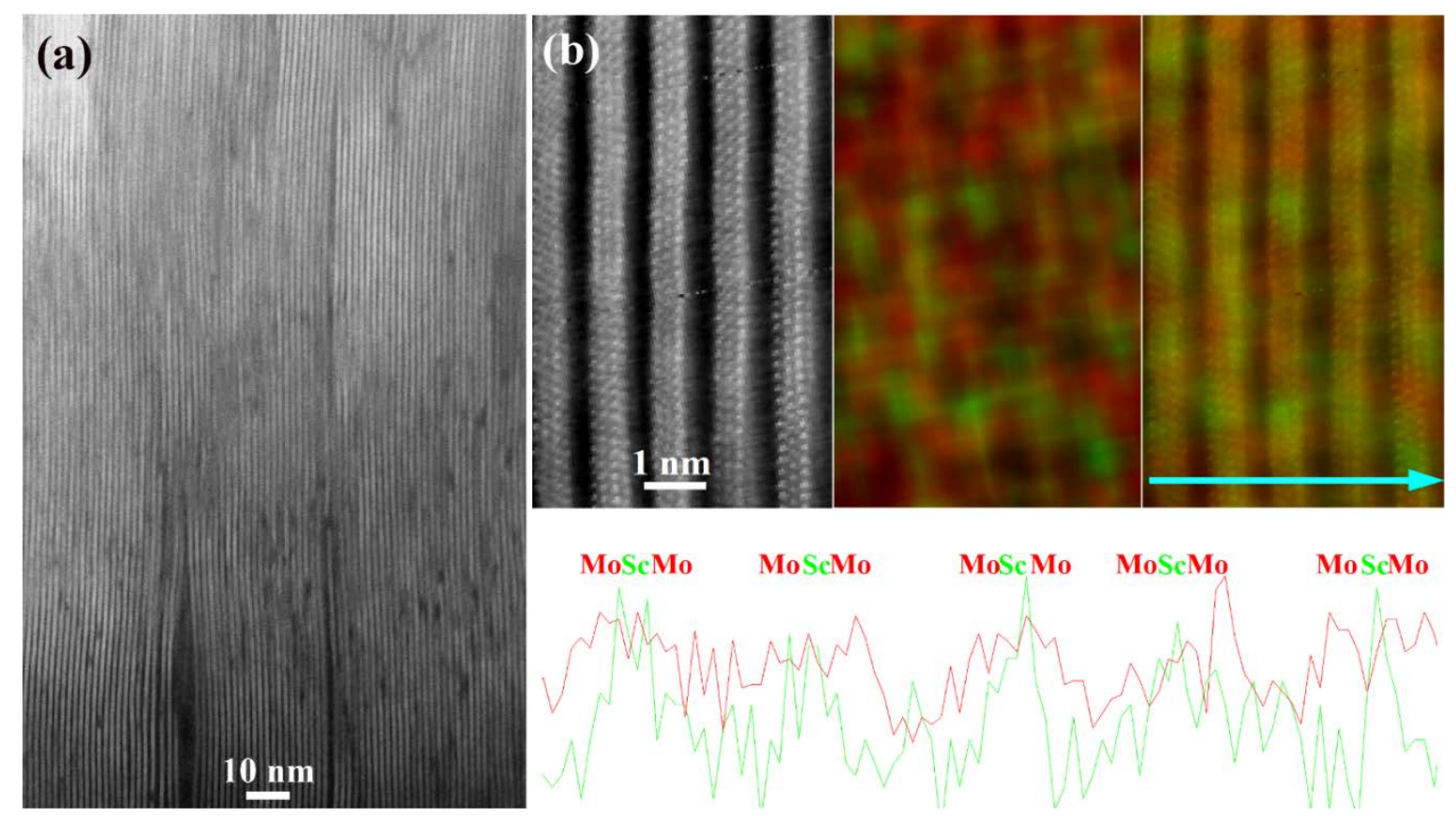

Fig. 4. (a) An overview STEM micrograph of MoScC MXene sample; (b) left: HRSTEM image, middle: the corresponding EDX map and the HRSTEM image superimposed with the EDX map; (c) a line scan of the EDX map shown by the above light blue arrow. 


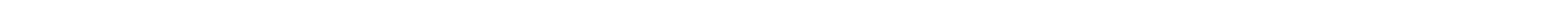




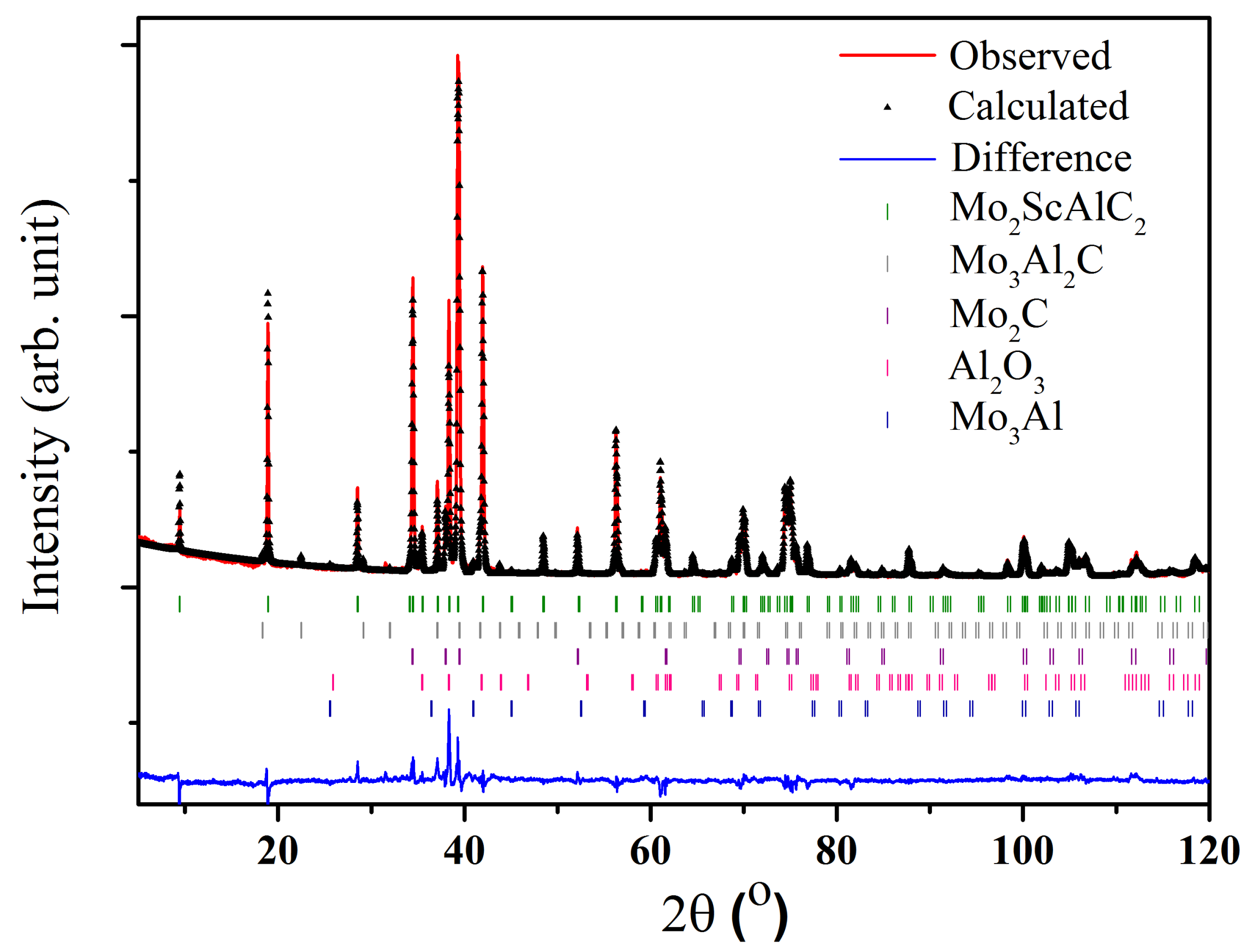


(a)

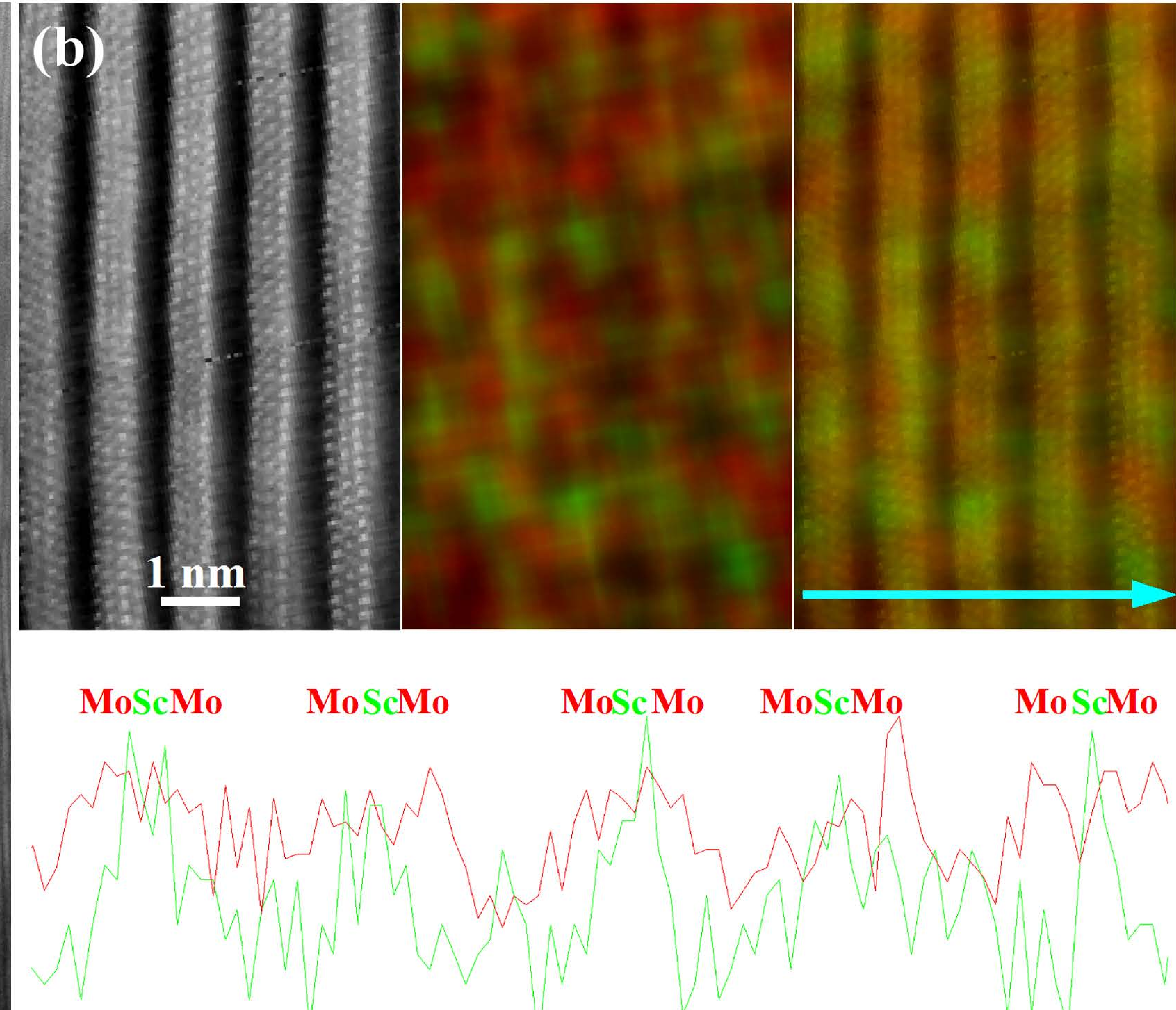




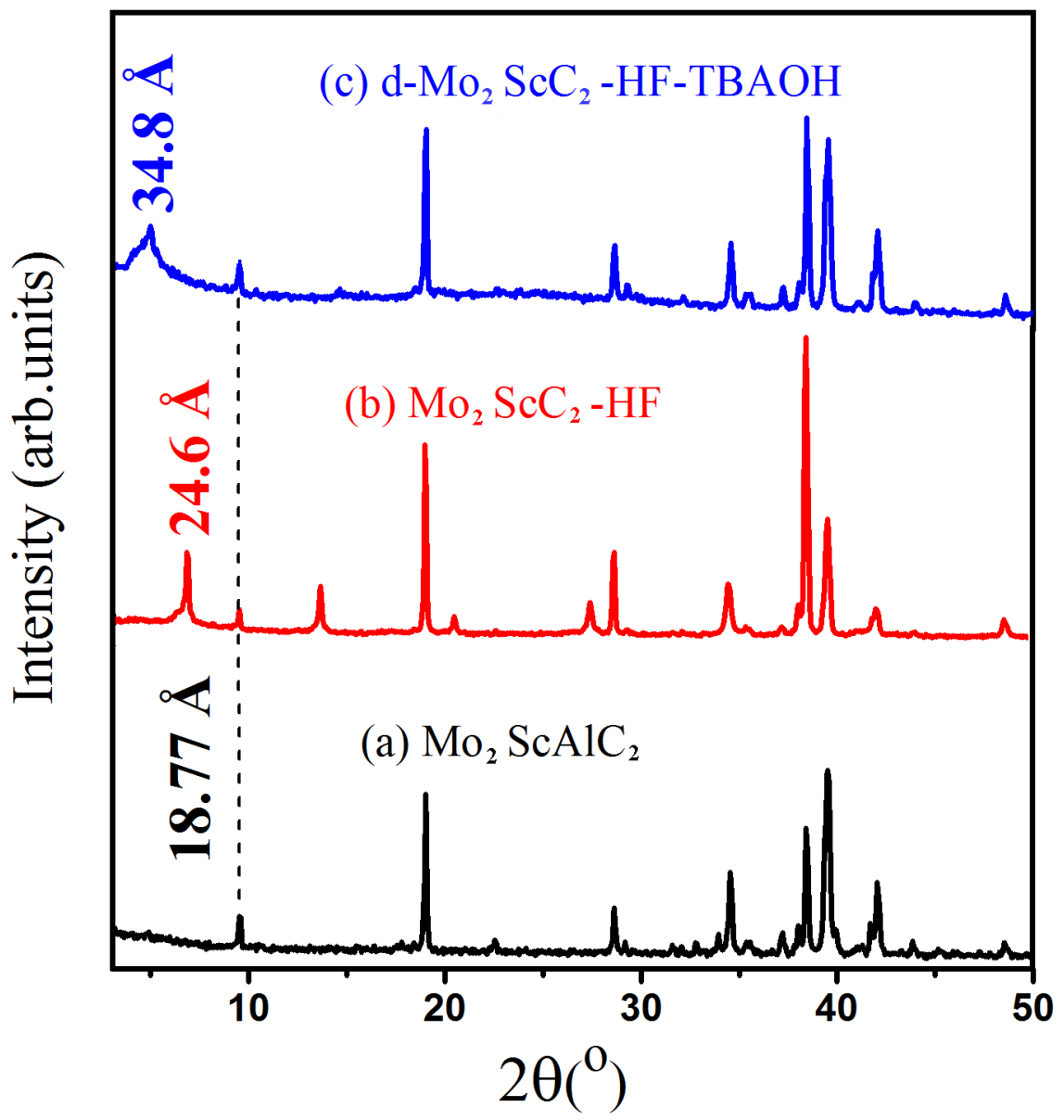

